\title{
Review on Femto-cell Networks Interference Management Techniques
}

\author{
Khalid Sheikhidris Mohamed ${ }^{* 1}$, Mohamad Yusoff Alias ${ }^{* 2}$, Mardeni Roslee*3 \\ * Centre for Wireless Technology, Faculty of Engineering, \\ Multimedia University, Cyberjaya, 63100, Malaysia. \\ ${ }^{1}$ khalidkaradh@hotmail.com \\ ${ }^{3}$ yusoff@mmu.edu.my \\ 2 mardeni.rolsee@mmu.edu.my
}

\begin{abstract}
Future cellular communication will relentlessly and incessantly hinge upon heterogeneous network due to its' capabilities in maintaining lofty capacity figures. It also strengthens the voice quality, accelerate data transmission, and optimize the users' battery consumption. However, its performance is often debatable due to the interference that may accompany its service. This paper focuses on studying the interference that affixes Femto-cells as an entity of heterogeneous networks due to its appealing functionalities and performance qualities. Moreover, the paper categorizes the types of interference based on the network platform and its source, discusses different contributions related to the topic based on schemes' efficiencies and complexity levels, and presents the most decent and stable scheme from the authors' prospective of view.
\end{abstract}

Keywords - Femto-cell, Interference management, Power calibration, Spectrum utilization.

\section{INTRODUCTION}

The term Heterogeneous (HetNets) Networks in wireless communication defines characteristically divergent networks in- terms of access platform and/or the cells' coverage areas such Micro-cells, Pico-cells, and Femtocells. HetNets will absolutely embody the future of Long Term Evolution (LTE) systems as it is expected to densely be deployed due to its' phenomenal capabilities of extending resources utilization, and the cutbacks of deployment costs to the service providers.

Additionally, each of the HetNets affiliates has some peculiar characteristics as shown in Table I to assort its' functionality and environment suitability.

TABLE I: Characteristics of different HetNets affiliates

\begin{tabular}{|c|c|c|c|c|}
\hline Cell Type & Coverage & Tx Power & Deployment & Access \\
\hline Macro & $\leq 35 \mathrm{~km}$ & $\leq 46 \mathrm{dBm}$ & Service provider & Cellular \\
\hline Micro & $\leq 2 \mathrm{~km}$ & $\leq 33 \mathrm{dBm}$ & Service provider & Cellular \\
\hline Pico & $\leq 200 \mathrm{~m}$ & $\leq 30 \mathrm{dBm}$ & Service provider & Cellular \& Wifi \\
\hline Femto & $\leq 10 \mathrm{~m}$ & $\sim 20 \mathrm{dBm}$ & Users & Cellular \& Wifi \\
\hline Relay & $\leq 200 \mathrm{~m}$ & $\leq 30 \mathrm{dBm}$ & Users & Wifi \\
\hline
\end{tabular}

On the other hand, service providers tend to advance networks' performance by geographically fixing cell-site locations in a way that guarantees the best spectrum utilization and maintains the finest Quality of Service (QoS). However, network engineers have no control on Femto-cell and Relay nodes locations as users privately deploy the entities wherever it is needed.

Consequently, few snags such as interference and resources' scarcity may surface resulting an adverse performance due to [1]:

- Increased number of cells deployed in the area.

- Close distance deployment.

In this context, interference can be categorized based on either the link channel or the resource tier. In the uplink channel, a user that is transmitting at high power level may cause interference to a neighboring cell. While in the down-link an indoor cell's power that is leaked through windows, balconies etc. may impede a nearby User Equipment (UE).

As shown in Figure 1a, cross-tier interference implies the interference that occurs in between entities that are associated to different tiers. Similarly, alike tier entities exchange Co-tier interference as shown in Figure 1b.

Additionally, considering areas that have less communication demands [2] and/or dead zones that may also emerge as a result of impenetrable infrastructures which by its turn inhibit UEs accessing network resources. Outage probability will cruelly inflate resulting drastic deterioration of the network QoS [3] [4]. 


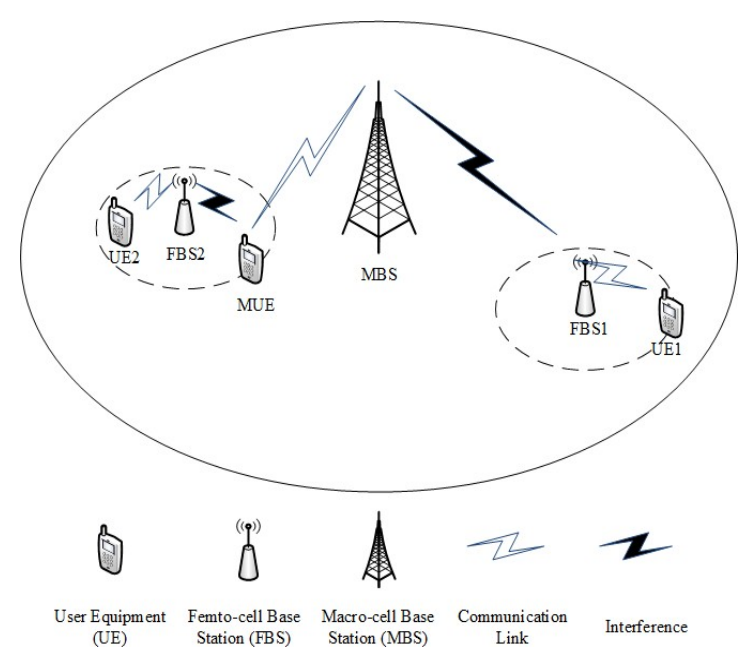

(a) Cross-tier Interference scenarios.

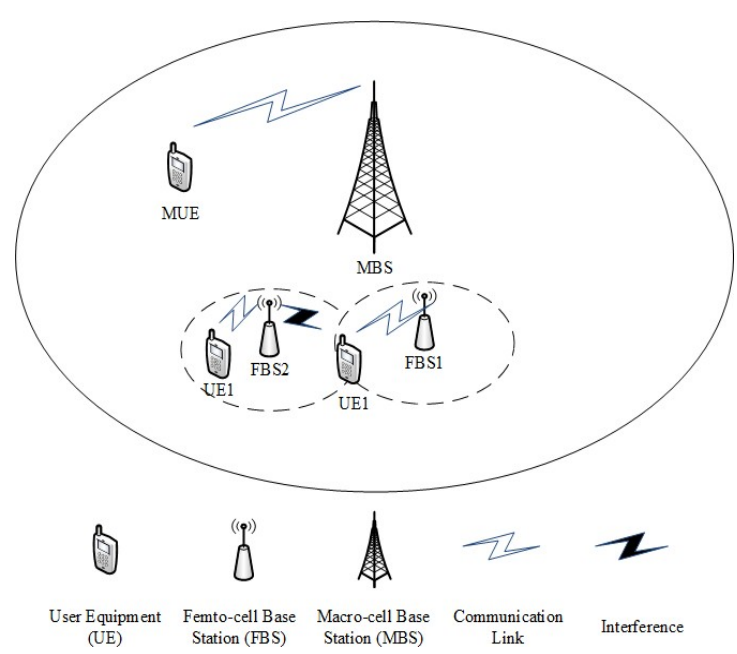

(b) Co-tier Interference scenarios.

Figure 1: Interference scenarios in hetnets

Hence, the notion of conventional Femto Base Station (FBS) or Home Node B (HeNB) in LTE emerged as a stand-alone entity that incorporates the functionality of the other HetNets' entities inside offices and small residential places [5] connecting cellular UEs to the core network via broadband connections.

Femto-cells reward UEs granted access to the network resources, enhance the voice quality, and reduce the battery consumption due to small distance between the FBS and the UE. Hence, it expand the network capabilities to an explicitly perfect scale. On top of that, FBSs are entirely mobile and can be installed by the users as it possess plug-and-play functionality. However, the interference generated by FBSs is explicitly high and enough to conversely enhance the QoS and network performance [6]. Predominantly, interference occurs as a result of power leaks from an aggressor to a victim. Hence, Femto-cells deliver interference to a nearby Macro-cell User Equipment (MUE) or a Femto-cell User Equipment (FUE) that are attached to another MBS or FBS, respectively in the down-link. And conversely, FUEs interfere with a nearby FBS or MBS in the downlink [7].

Eventually, the topic of resources and interference management has manifested and enticed many engineers and researchers over the past few years. One of their most evident solutions is to land more resources to be allocated for Femto-cells and forestall the co-tier interference. However, this was instantly discarded due to increased costs of buying extra resources. Alternatively, granting FBSs to reuse the MBS spectrum was another conspicuous solution. However, it was also non-realistic as it diminishes the consequences of the tremendous amount of resulting interference due to small distances in dense deployments.

Accordingly, several contributions adhered power calibration mechanisms as the safest solutions specifically in dense deployment areas as reducing the FBSs transmission power certainly lessens both co-tier and cross-tier interference [8]. However, it may indirectly disturb edge users' received signal quality resulting either complete outage or meager reception quality. Additionally, edge users are mostly vulnerable to experience higher levels of interference from nearby entities when receiving poor quality signals [9].

Eventually, more complex schemes were developed to clear the argument on interference such as [10] who proposed deploying several antennas in multi-floors building to seemingly curb down interference in different levels. The authors of [11] also proposed a queuing algorithm to uphold the system capacity at peak hours. The algorithm schedules the resource block, marks it with a flag, queues the users, and associate users with resources respectively. However, the scheme did not consider dual allocation probability which may unintentionally generate additive interference and further degradation of the QoS.

This work is considered as an affluent study of Femto-cell interference management which encloses a discussion of different schemes with regards to the interference environment. The remainder of this paper is organized as follows: In Section II, we discuss co-tier interference management related schemes. Cross-tier interference management schemes are to be discussed in Section III. Section IV discusses general interference management schemes. We finally conclude the paper in Section V. 


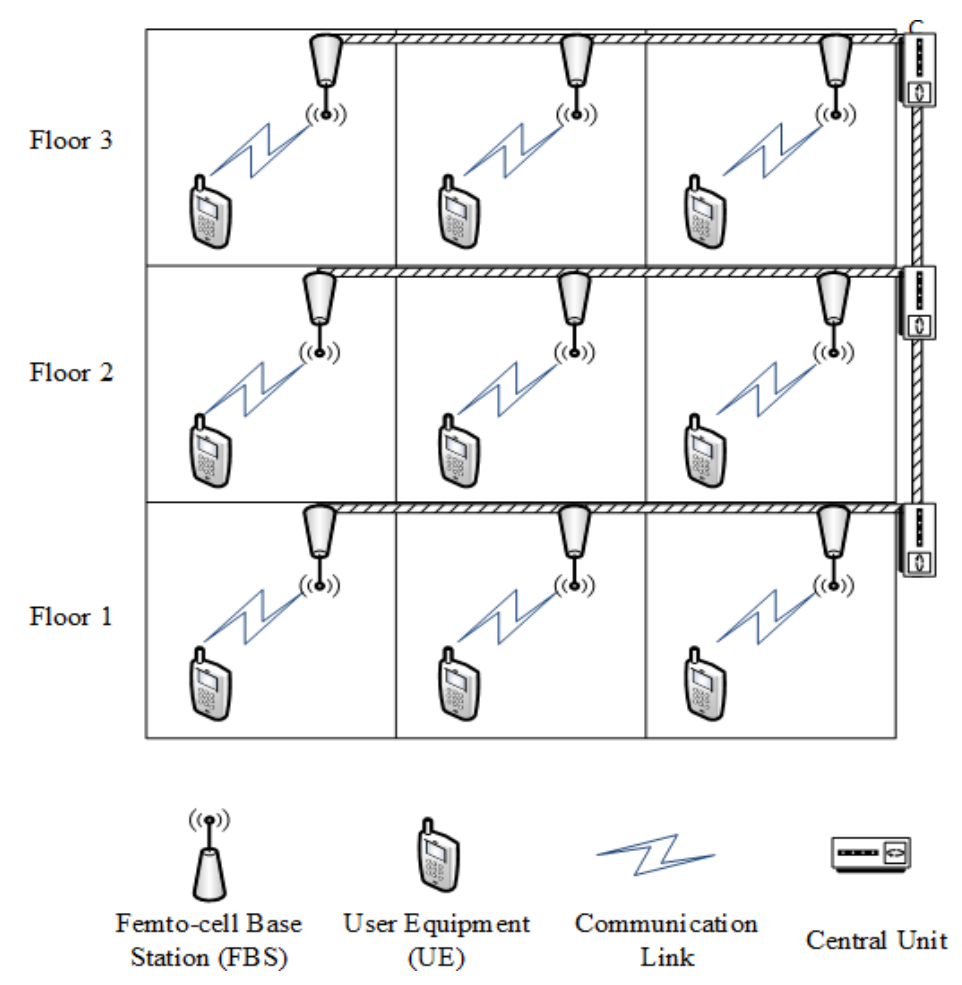

Fig. 2: Distributed Antenna Systems scenario in multi-floor building.

\section{CO-TIER INTERFERENCE MANAGEMENT}

Co-tier networks offer relatively increased capacity figures with a finite spectrum in spaced deployment networks [12]. However, throughput levels may often deteriorate as a result of outage probabilities in arbitrary deployed networks such as Femto-cell networks. Instead, 3rd Generation Partnership Project (3GPP) LTE offered FBSs to [13]:

1) Share the same spectrum orthogonally.

2) Use sub divided spectrum bands.

3) Orthogonally utilize a portion of the spectrum.

The following sub-sections discuss the various schemes that oversee co-tier interference in cellular Femto-cell networks.

\section{A. Power Management Schemes}

Authors in [14] proposed to deploy a Distributed Antenna System (DAS) connected to several FBSs in a multi-floors building to mitigate interference. Each FBS is further connected to a Central Units (CU) that performs the mitigation process upon decoding interfering signals. Consequently, other CUs receive an information bit to reconstruct and subtract the interfering signals as well.

To understand this method, let us assume a building with several floors as shown in Figure 2. The number of CUs is determined by the number of floors and the number of mobile stations that receive and transmit in the same frequency is denoted by $U$. The desired $\mathrm{CU}$ in which operates the desired user applies interference cancellation and exchange the information with the other CUs. The channel impulse response should be known to all other CUs so that it is able to perform the cancellation. The impulse response of a user $u$ in the $v$ th floor is calculated by the following equation:

$$
h_{u, v}(d)=\left(d_{u, v}\right)^{-\lambda u, v} \alpha_{u, v} \exp ^{j \theta_{u, v}} \delta(t)
$$

where the channel fading factor is denoted by $\alpha_{u, v}$, phase coefficient is denoted by $\theta_{u, v}$, transmission path loss is $\left(d_{u, v}\right)^{-\lambda_{u, v}}$, floor attenuation factor is $\lambda_{u, v}$, and the distance between the user and the FBS is denoted by $d_{u, v}$. Eventually, the received signal by the desired user $u^{\prime}$ can be calculated through Equation 2 [14]:

$$
\begin{gathered}
r_{u^{\prime}}(t)=x_{u^{\prime}}(t) \otimes h_{u^{\prime}, v^{\prime}} t+\sum_{u \neq u^{\prime}}^{U} x_{u^{\prime}}(t) \otimes h_{u^{\prime}, v^{\prime}}(t)+\eta(t) \\
=x_{u^{\prime}}(t) \cdot\left(d_{u^{\prime}, v^{\prime}}\right)^{\lambda} u_{u^{\prime}, v^{\prime}} \alpha_{u v^{\prime},}(t) e^{j \theta_{u, v}(t)}+ \\
\sum_{u \neq u^{\prime}}^{U} x_{u}(t)\left(d_{u^{\prime}, v^{\prime}}\right)^{\lambda}{ }_{u^{\prime}, v^{\prime}} \alpha_{u^{\prime}, v^{\prime}}(t) e^{j \theta_{u, v}(t)}+\eta(t)
\end{gathered}
$$


where $\eta(t)$ denotes the received Additive White Gaussian Noise (AWGN) at the FBS. The equation consists of the interference contribution of the $u^{\prime}$ user at the first part and the interference contribution of the co-stations at the second part.

If a CU detects any interference at any FBS, the next FBS reconstructs the interference and subtract it from the received signal. Equations 3, 4, and 5 calculate Signal to Interference Plus Noise Ratio (SINR), the residual interference from a user, and the reduced interference SINR, respectively [14]:

$$
\begin{gathered}
\gamma=\frac{E\left[S_{u}^{2}\right]}{\sum_{u \neq v}^{U}\left(P_{s \cdot} \cdot\left(d_{u^{\prime}, v^{\prime}}\right)^{-2 \lambda_{u, v}}+\sigma_{u}^{2}\right)} \\
Y=\sqrt{P_{s}} b_{u^{\prime}}\left(d_{u^{\prime}, v^{\prime}}\right)^{\lambda} u_{u^{\prime}, v^{\prime}} \alpha_{u, v,}(t) e^{j \theta_{u, v}(t)}+\sum_{u \neq v}^{U} \Delta l_{u, v^{\prime}}+\eta \\
\gamma=\frac{E\left[S_{u^{\prime}}^{2}\right]}{\sigma_{\Delta l\left(v \prime^{\prime}\right)}^{2}+\sigma_{\eta}^{2}}
\end{gathered}
$$

The scheme offers a remarkable reduction of interference and improved throughput levels. However, the system encompasses high complexity structure and relatively increased installation cost.

Alternatively, [15] proposed a Dynamic Transmitter Gain Management (DTGM) scheme in an enterprise Femto-cell deployment as in Figure 3. The authors proposed a virtual Femto-cell Management System (FMS) to be employed in-order to control FBSs' transmitter gain and further mitigate the interference and improve the overall SINR. The FMS first categorizes each FBS based on its location and tier (Center FBS and tier FBS), then it collects the SINR readings at all FBSs. If the SINR of a center FBS is below the threshold, the FMS sums the entire tier SINRs and takes the average to tune the center FBS to the new gain. The SINR of all FBSs is calculated through Equation 6.

$$
S I N R=\frac{P_{f} \times G_{f}}{N \times \Delta f+\sum\left(P_{F} \times G_{F}\right)}
$$

where $P_{f}, P_{f}$, and $\Delta f$ are the Femto-cell transmission power, transmitter gain, and the frequency shift, respectively.

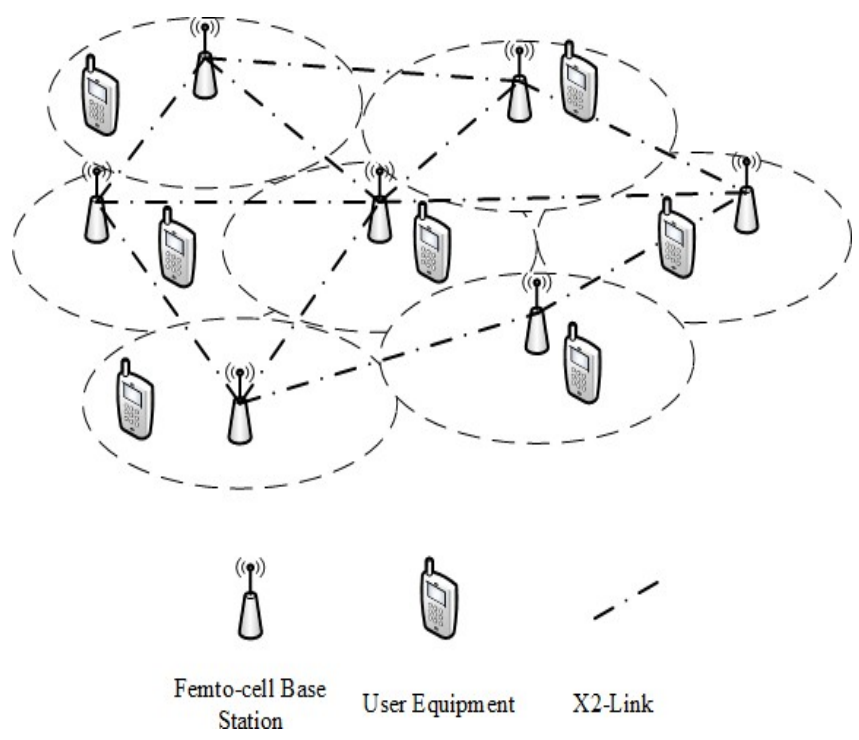

Fig. 3: Enterprise Femto-cell Deployment [15].

Aside from that, [16] proposed to divide the Femto-cell coverage area into two regions namely inner and outer, whereby initially the outer region width equals to zero until the Channel Quality Identifier (CQI) level degrades below the CQIT hreshold. Users send frequent CQI readings to the FBS, the FBS later assigns higher modulation scheme to users bearing CQI levels. Furthermore, it allocates different resources to the outer region than the inner region in a way that neighboring FBSs use distinctive resources formation. However, closely deployed Femto-cells cause the inner region to aggressively shrink and hand- off users to the outer region which may baffle the resource association process.

Similarly, [17] proposed to adjust the transmission power based on analyzing the Received Signal Strength Indicator (RSSI) and the effect of interference on FBSs' SINR. A Femto-cell gateway later groups FBSs based on the interference exchanged between neighboring Femto-cells. It then determines the minimum of the orthogonal sub-channels needed to provide the targeted performance with a decreased interference. 
Comparing the previous schemes, we find that DAS performance grants the system with up to $13 \mathrm{~dB}$ gain of SNR due to the reduced interference. However, SNR levels deteriorate when the number of interferers increase because of the relatively high estimation errors due to the small distance between the FUEs and the FBSs. Subsequently, the DTGM method achieves only $2.17 \mathrm{~dB}$ improved SNR gain while it improves the data rate by $11 \%$ with the same FBS coverage. Lastly, the scheme proposed in [17] improves both SNR and throughput by $27 \%$ as well as the spectral efficiency allowing the system to accommodate higher number of UEs.

\section{B. Spectrum Management Schemes}

The authors of [18] proposed a robust spectrum sharing technique that allows FBSs to reuse nearby MUEs resources to avoid co-channel interference in between Femto-cells. The Femto-cell listens to the Macro-cell's down-link and at the same time detects spectrum opportunities at the up-link. The Femto-cell will be able to discover the Macro-cell bandwidth so that it is able to prevent any overlapping to occur. However, in the case of any overlapping, the FBS will further sense MUEs presence in the up-link and set a specific threshold whereby if the threshold is high then the Femto-cell selects the nearby MUEs resources and the opposite is also true. On the other hand, each MUE has a unique identifier known as the Cell Radio Network Temporary Identifier (CRNTI) used to identify the MUE resources. Hence, upon selecting the MUE, the Femto-cell acquires that specific C-RNTI and uses it to locate the resources on the MBS down-link.

[19] proposed to divide both the MBS coverage area and the spectrum into smaller regions and portions, respectively. Eventually, all MBS center zones will have frequency reuse factor 1 and allocate a unique set of resources to the edge zones. FBSs will further calculate the received power from all neighboring cells and estimate SINR and throughput. This maximizes the throughput and prevents the interference between the MBS and FBS. However, the FBS resource allocation process is random and neither rely on any algorithm nor consider the distance in between FBSs itself which may result in additional FBS-FBS interference.

Additionally, [20] proposed a scheme that allocates Femto-cells with certain orthogonal frequency using a Femto-cell Network Controller (FNC) that is connected to numerous FBSs. The FNC acts as a virtual MBS to the core network and as a virtual core network to the Femto-cells. It also has the knowledge of all FBSs' locations of the cell as well as the capability of adjusting the transmit power and shuffling the resources of all FBSs. The scheme categorizes the serving FBS into closed and open-access entities whereby only the openaccess FBSs coverage area is divided into inner and outer regions. The allocated frequency of the closed-access FBS is reused into the inner regions of the open-access FBSs. Similarly, the frequency of the outer region is eventually allocated to the most far ones. The proposed scheme increases the spectral efficiency and decreases the interference probability amazingly. However, scenarios of having all FBS as open or all closed-access entities over distance variations of both FBS and FUE were not considered.

Similarly, [21] categorized the users into Guaranteed Bit Rate (GBR) and Variable Bit Rate (VBR) users authorizing GBR users to access resources of the neighboring cells and later on release interference free resources to be used by VBR users. This achieves higher bit rate values and exploits the frequency reuse by using a Successive Group Decoder (SGD). To understand the scheme, let us assume neighboring Femto-cell $F B S_{1}$ with a GBR user $U E 1_{G B R}$ and $F B S_{2}$ with a VBR user $U E 2_{V B R}$ in a grid deployment environment. $F B S_{1}$ assigns frequency to its user until the rate requirements are satisfied and the rest of the resources are to be assigned to the VBR users. Now, $\mathrm{UE}^{2} \mathrm{~V}_{B R}$ is only allowed to access the same resources only if the decoding rate of $U E 2_{V B R}>U E 1_{G B R}$.

Alternatively, [22] introduced a scheme that considers using a Macro-cell frequency scheduling information for the spectrum sensing purposes in-order to avoid interference between FBSs and nearby MUEs in both uplink and down-link. Moreover, it prevents FBSs from using the preoccupied resources by the Macro-cell users. It also calculates the sampled signal in time domain and superposition the aggregate received signal from all users through the following:

Sampled $_{n}=\sqrt{P_{t x, i}} \sum_{k \in \Gamma} X_{i}(k) \exp \left(\frac{j 2 k n}{N}\right)_{-\mathrm{N}_{\mathrm{cp} \leq \mathrm{n} \leq \mathrm{N}-1}}$

where $P_{t x, i}$ is the transmission power for user $i$, the sub-carrier index is $k$, Gamma is the total set of subcarriers, and $N_{c p}$ is the length of cyclic prefix and the data of the user is $X_{i}(k)$.

Equation 7 arrives with a time delay that can be calculated through Equation 8.

$$
\delta_{i}=\left[\frac{N_{\tau_{i}}}{T}\right]
$$

where $\tau_{i}$ is the propagation delay and $T$ is the duration of the data in the signal. Moreover, the aggregate received signal with AWGN in time domain is calculated by:

$$
y(n)=\sum_{i}^{N_{n}} y_{i}(n)+\omega(n)
$$


Thus, the proposed scheme can be summarized in Figure 4 which illustrates the different arrival times of the signal and its effects. Figures (a) and (d) show the structure of the symbol, (b) shows the arrival periods from different users that are within the cyclic prefix period while (c) shows the effects of arrival times that are greater than the cyclic prefix periods.

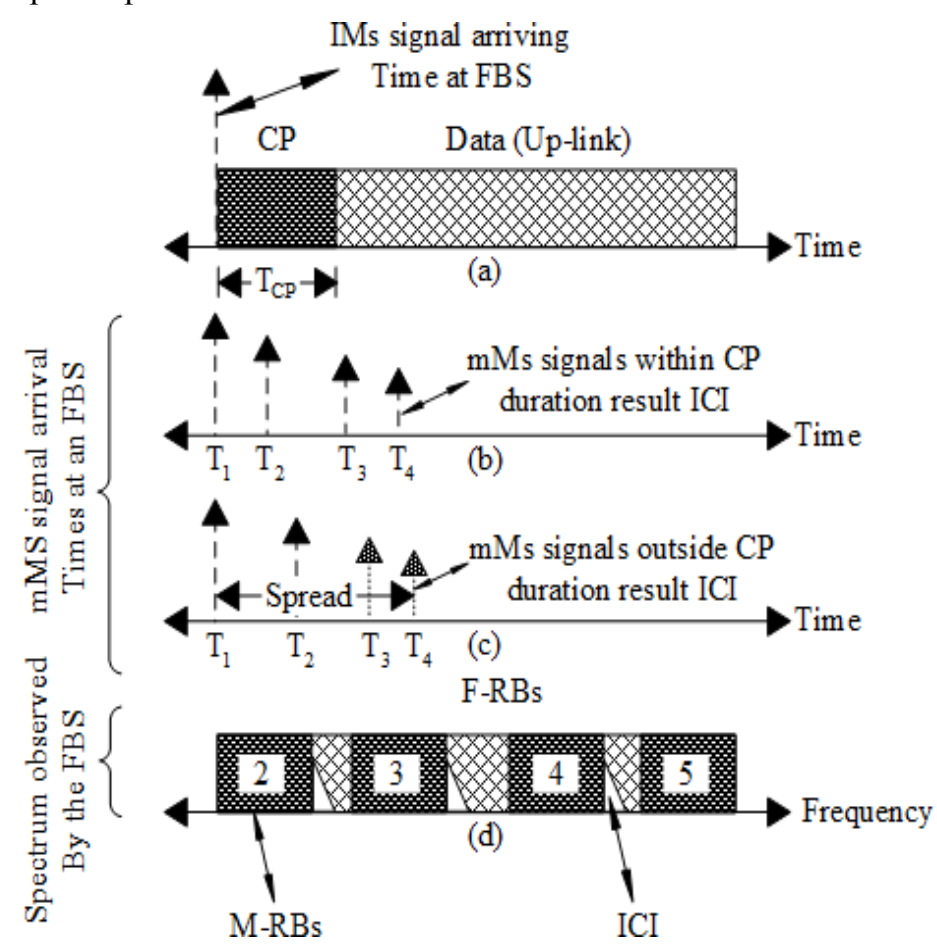

Fig. 4: Illustration of the Macro-cell users signal arriving at different timing [22].

The throughput in [18] scheme granted all users approximately additional 10 Mbps. However it has only accommodated 5 users. Alternatively, [21] accommodated 10 users granting each approximate gain of $40 \%$. Regardless of this, when considering congested environments, the throughput in both schemes is unpredictable and vague. Subsequently, [19] maximized the system capacity and achieved more stable and predictable performance of up to 20,40, and $60 \mathrm{Mbps}$ for 10,20, and 30 users, respectively.

\section{Summary}

In summary, spectrum management schemes seem to be more preferable over power management schemes in-terms of decreased complexity levels and cost efficiency. It also scores high capacity figures and relatively improved levels of throughput.

TABLE II: Comparison of existing interference reduction techniques [23] [24]

\begin{tabular}{|l|l|l|}
\hline Scheme & Pros & Cons \\
\hline $\begin{array}{l}\text { Spatial Channel } \\
\text { Separation }\end{array}$ & $\begin{array}{l}\text { No coordination nor Feedback is } \\
\text { needed between Macro } \leftarrow \text { Femto }\end{array}$ & - \\
\hline Coordinated Scheduling & - & Coordination is difficult \\
\hline No Coordination & - & Radio link failure \\
\hline Fixed Power & Easy and simple to implement & $\begin{array}{l}\text { The surrounding environment doesnot have } \\
\text { any effect on it, thus, interference still exist }\end{array}$ \\
\hline Adaptive Power Setting & $\begin{array}{l}\text { Edge FBSs experience less } \\
\text { interference }\end{array}$ & High interference when large power offsets \\
\hline
\end{tabular}

\section{CROSS-TIER INTERFERENCE MANAGEMENT}

In discussion of interference management in cross-tier Femto-cell networks, it seem to be more challenging than in co-tier networks due to the chaotic deployment and resource allocation of resources which complicate interference prediction and later management. However, it is possible to mitigate the interference via FFR, spatial channel separation, power calibration etc. Accordingly, [23] and [24] provided surveys on the mentioned mechanisms as summarized in Table II.

Moreover, the subsequent sub-sections review multiple cross-tier interference management in Femto-cell networks schemes. 


\section{A. Power Management Schemes}

The authors of [25] had proposed an improved version of the conventional Code Division Multiple Access (CDMA) Femto- cell networks that reduces the cross-tier interference by allowing FUEs and MUEs to cooperatively share the same spectrum. However, MUEs are prioritized to access resources over the FUEs. Additionally, MUEs' QoS is maintained by employing a joint power and admission control technique and further exploit the remaining spectrum to be used by FUEs. The prioritization of UEs is achieved depending on the user's SINR threshold and QoS requirements with respect to the access medium.

Additionally, after calculating UEs' SINR and the received interference from both Macro-cell and neighboring FBSs, the scheme repeatedly evaluates SINR levels to update the iterative SINR $p_{i}$ as in Equation 10.

$$
P_{i}(t+1)=\gamma_{i}(y) \frac{I_{i}(t)}{g_{i}(t)}=\frac{\gamma_{i^{\prime}}(t)}{\gamma_{i}(y)} p_{i}(t)
$$

where $\gamma_{i}$ is the actual SINR and $\gamma_{i}{ }^{\prime}$ is the SINR threshold and $p_{i}$ is the transmission power.

A negligible amount of SINR helps nothing but rather generates un-necessary interference to FUEs.

Similarly, [26] proposed a scheme that employs a hierarchical game theory between the authorized higher multi-agents, MUEs and the non-authorized lower multi-agents, FUEs. MUEs and FUEs compete among themselves in a non-cooperative manner to maximize their iterative SINR and continuously look forward for the other multi-agents response. On the contrary, FUEs adjust their iterative transmission power in response to MUEs' iterative power that as in the following:

$$
\begin{gathered}
P_{t}^{M}(t+1)=\min \left\{\left[\frac{I_{i}\left(p_{-i}\right)(t)}{G g_{i}^{M}} \cdot \gamma_{i}^{M *}(t)\right]^{+} \cdot P_{i}^{M_{\max }}\right\} \\
P_{t}^{F}(t+1)=\min \left\{\left[\frac{I_{n}\left(p^{F}-n\right)\left(\Gamma_{n}^{F}\right)}{G g_{n . n}^{F}} \cdot \gamma_{i}^{M *}\right]^{+} \cdot P_{n}^{F_{\max }}\right\}
\end{gathered}
$$

where $G g$ is the gain and $\Gamma$ is the required SINR.

Accordingly, Figure 5 presents a comparison of the estimated iterative SINR of both MUEs and FUEs in [25] and [26]. Additionally, Figure 6 presents another comparison of the average estimated interference contribution from both MBS and FBS in [25] and [27]'s scheme. Consequently, FBSs set initial transmission power to FUEs and continuously update it through the following equation:

$$
P_{t}(t+1)=\min \left\{\frac{I_{t}\left(p_{-i}\right)(t)}{g_{i, i}(t)}\left[\gamma_{i}+\frac{1}{\alpha_{i}} \ln \frac{\alpha_{i} g_{i, i}(t)}{b_{i} g_{i}(t)}\right]^{+}, P_{\max }^{n}\right\}
$$

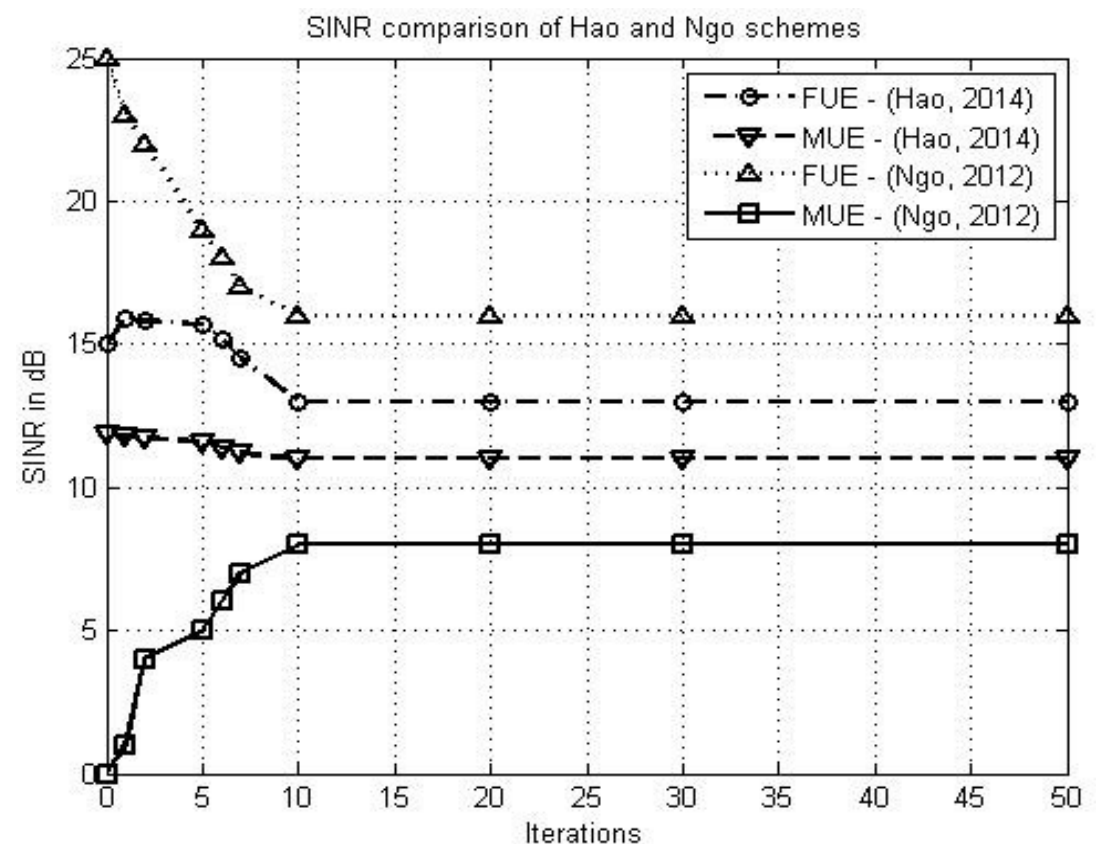

Fig. 5: Comparison of the estimated iterative SINR of both MUEs and FUEs in [25] and [26]. 


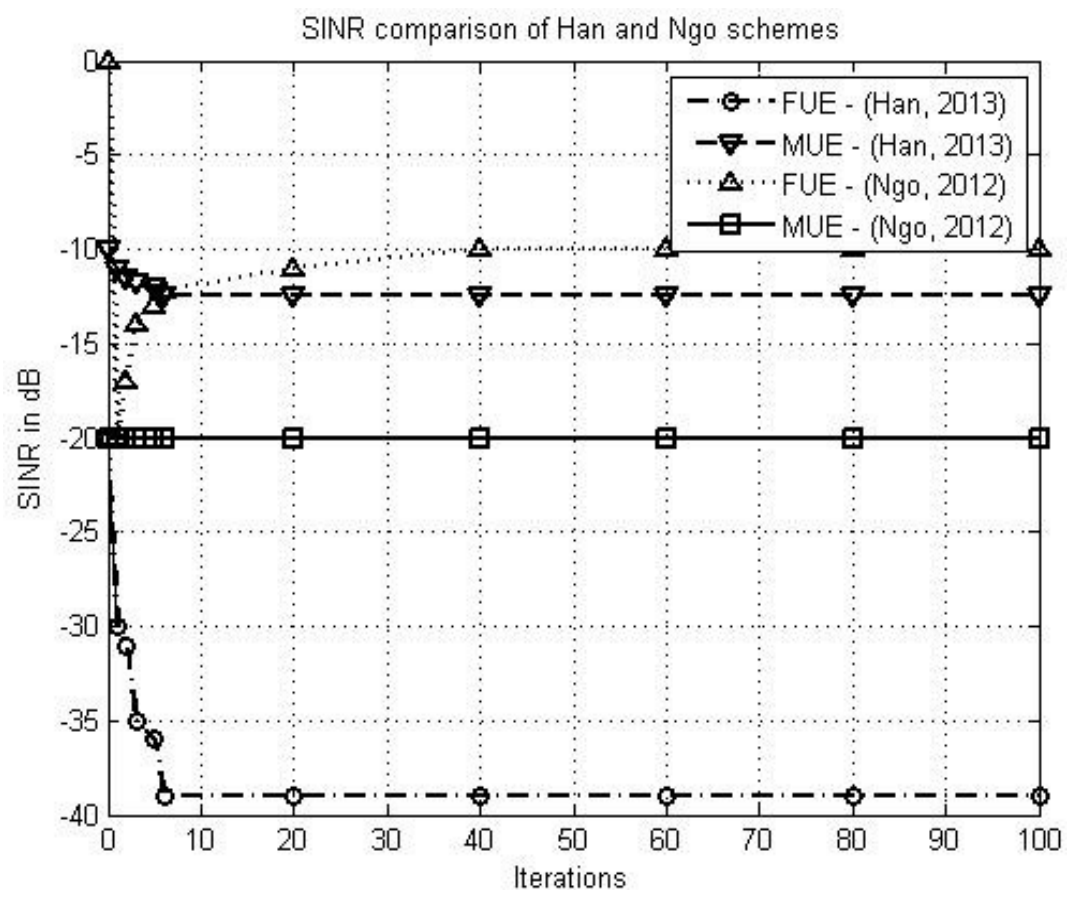

Fig. 6: Comparison of the estimated iterative transmission power of both MUEs and FUEs in [25] and [27]

\section{B. Spectrum Management Schemes}

Cellular systems' resource efficiency solely depend on systems' capability to utterly utilize the available spectrum and the ability to accommodate large number of users without degrading any of their QoS requirements. With regards to this, [28] proposed a scheme for a grid deployment OFDMA Femto-cells downlink. The scheme forces interfering MUEs to occupy a certain allocated sub-carriers, and the non-interfering MUEs and FUEs to use different sets of sub-carriers. Furthermore, the scheme employs a power adaptation algorithm to calibrate the sub-carriers' transmission power.

Furthermore, an MUE is categorized as dedicated user if the MUE shares resources with an FUE, otherwise it is a regular user. Consequently, it is prohibited from sharing the resources with other dedicated users.

The authors, illustrated the outcomes of the scheme in a scenario of two tier network whereby on MBS and a grid of adjacent Femto-cells is considered. If an MUE and an FUE share the same resources and an FBS interfere with an MUE, that MUE is classified as a dedicated user, else-wise it is a regular user and prohibited from sharing the spectrum with the dedicated users. The resources allocation is based on the minimum transmit power that results from the following model:

$$
\begin{gathered}
\min _{\text {power }} \sum_{m=1}^{M} \sum_{n=1}^{N} P_{m n} \\
C_{1}: \sum_{n=1}^{N} r_{m n} \geq R_{m n}^{m}, \forall m \\
C_{2}: \frac{p_{m n}|| a_{m} h_{m n} \|^{2}}{\sigma^{2}} \geq \gamma_{\mathrm{mn}}, \forall m, n \\
C_{3}: p_{m n}=0, \forall m \in \text { lambda } a_{\text {dedicated }}, n \in \omega_{f}
\end{gathered}
$$

where $\omega_{f}$ is the sub-carriers set, $C_{1}$ is the sum rate, $C_{2}$ is SINR, $C_{3}$ is the dedicated resources that cannot be shared.

Alternatively, [29] employed a frequency allocation scheme that has two branches. The first divides the entire bandwidth $B$ into three sub-bands $B_{1}, B_{2}$, and $B_{3}$, the coverage area into three sectors $S_{1}, S_{2}$, and $S_{3}$ and allocates each band to a specific sector. Furthermore, if a Femto-cell is being deployed in $S_{1}$ that is served by frequency band $B_{1}$, it is assigned any of the other two frequency bands $B_{2}$ or $B_{3}$.

The second branch divides the area covered by an MBS into two different regions (center and edge) and similarly, the frequency into three bands. Hence, Femto-cells that exist in the center region are assigned the entire frequency but only during the first time slot of an LTE frame, while the edge Femto-cells are assigned the second time slot with 3 times the transmission power of the Femto-cells that exist in the inner region. The proposed scheme offered the following: 
- Reduced Macro $\rightarrow$ edge-Femto interference because of the distance.

- Negligible edge-Femto $\rightarrow$ Macro interference due to the Femto-cells low transmission power.

- Interference only at the first time slot of the LTE frame for the Macro $\rightarrow$ Center-Femto.

- Reduced interference by $1 / 3$ as transmission happens only in ${ }^{1}$ of the bandwidth.

Additionally, the scheme also evaluated the system fairness to all users to receive $\operatorname{SINR}^{>} \operatorname{SINR}_{T}$ hreshold and showed substantial improvement. However, SINR level may decay when considering limited resources system.

Similarly, [30] proposed a scheme that processes the interference depending on the link and further divides the entire spectrum $D$ into four non-overlapping equal parts $D_{1}, D_{2}, D_{3}$, and $D_{4}$. It also divides the Macro-cell into inner (with a radius of $r_{\text {min }}$ ) and outer region which is $r_{\text {mout }}=r_{M B S}-r_{\text {min }}$ and three sectors $S_{1}, S_{2}$, and $S_{3}$ for the outer region. Whereas FBSs are only divided inßo three sectors $S \dot{1}, S \dot{z}$, and $S \dot{j}$, each is $\frac{2 \pi}{3}$. The four frequency parts are initially assigned to the Macro-cell three sectors and the inner region. However, FBSs utilize the three frequency sets that are assigned to the Macro-cell sectors. Hence, the inner region is using a unique set of frequency.

Subsequently, the same scenario is implemented for the up-link with a reversed mechanism. FBSs are divided into inner (with a radius of $r_{f}$ in) and outer region which is $r_{f o u t}=r_{F B S}-r_{f i n}$, and three sectors for the outer region, while the MBS is divided to only three sectors. Consequently, the interference is mitigated as MBS and the FBS regions are spaced apart. The design offers improved interference levels and an enhanced fairness for all the BSs to have shares of resources as compared to the previous scheme. However, it degrades MBS resource utilization capability and increases both the operational complexity level and manufacturing cost of the Femtocells.

Although the previous scheme generalized the topic of interference mitigation, [28] had presented a strong scheme that achieves approximately $18 \mathrm{~dB}$ of SINR gain and relatively improved average throughput.

\section{Interference Alignment and Coordination Schemes}

[31] proposed a cooperative interference alignment algorithm for two-tier Femto-cell networks which consists of two stages. The first stage determines and maintains SINR for MUEs while performing the interference alignment to mitigate the MBS interference contribution to the FBS. The second stage allows Femto-cells to determine the precoding vectors for the FUEs to maximize the sum-rate. The algorithm offered an increased Femto-cell throughput to relatively limited number of Femto-cell which limits the design's expected outcomes in dense areas.

Similarly, [32] proposed a successive semi-definite programming relaxing algorithm for interference alignment to minimize the sum Minimum Square Error (MSE) of FUEs which requires a minimal cooperation between MUEs and FUEs. However, the complexity of the design increases when employing a zero-forcing precoder to cancel the rest of the interference at each of the Femto-cells.

[33] proposed a scheme that exploits interference coordination by employing high tech interference aware receivers. Alternatively, it divides the spectrum into sub-carriers that are $15 \mathrm{KHz}$ each (12 sub-carriers form Resource Blocks in LTE) and considers a proportional fair scheduling algorithm to assign sub-carriers to the Femto-cells when needed. The received interference is calculated using the following:

$$
I_{u, b}=\Gamma_{f, m} \frac{P_{m, b} G_{m, u}}{L_{m, u}}
$$

where $\Gamma_{f, m}$ is 1 if the MBS and the FAP use the exact same group of resource blocks and 0 if not. The MBS power is

$P_{m, b}, G_{m, u}$ is the antenna gain, $d$ is the separation distance, and $L m, u$ is the overall channel loss. Furthermore, the loss can be calculated by:

$$
L_{m, u}=\frac{Z_{m, u}}{\omega \gamma_{m, u}^{\phi}}
$$

where $\gamma_{m, u}^{\phi}$ is the spacing between the MBS and the FBS, penetration loss is $\omega, Z_{m u}$ is a random variable, and $\varphi$ is the exponent of decay.

The scheme shares the spectrum between MBS and FBS to entirely eliminate the cross-channel interference. FBSs use relatively smaller portion of the spectrum while the MBS utilize the entire bandwidth. However, this will result an overlap in the spectrum usage (cross-tier interference) as in Figure 7. 


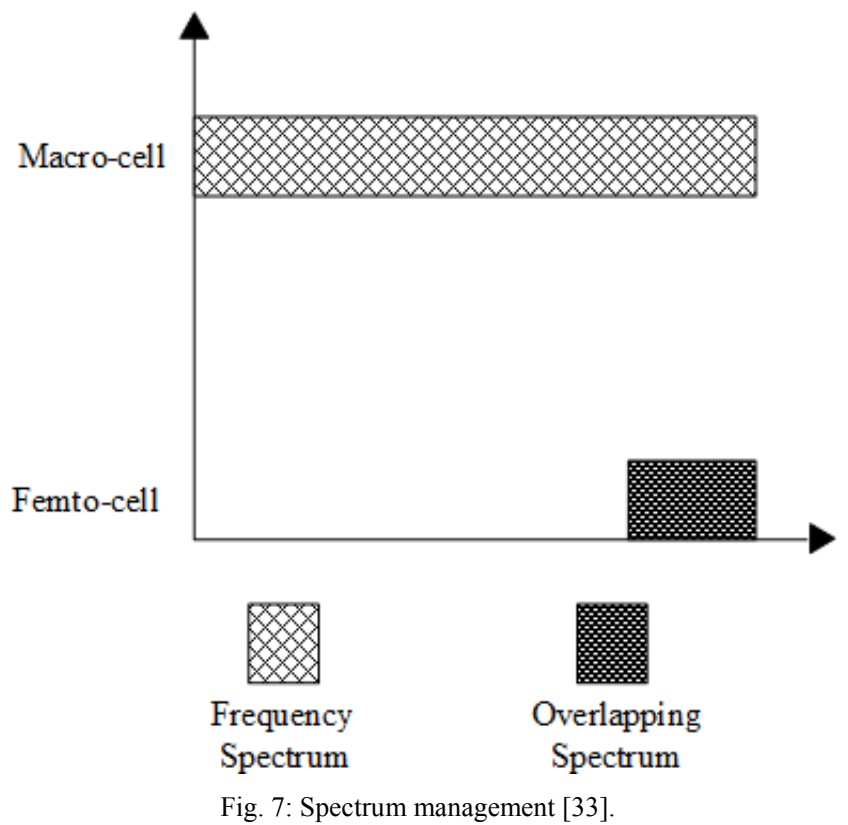

The MBS calculates the Signal to Interference Ratio (SIR) and SINR through the following equations:

$$
\begin{aligned}
S I R_{u, b} & =\frac{I_{u, b}}{P_{f, u} G_{f, u}} \\
S N R_{u, b} & =\frac{P_{f, u} G_{f, u}}{N_{0}}
\end{aligned}
$$

where $N_{0}$ is the thermal noise.

Eventually, calculating SIR in advance effectively helps to allocate the resources and further exploits the interference by the MBS presenting a sum rate of approximately $40 \mathrm{Mbps}$. Consequently, reliable utilization of resources and reduced interference levels.

\section{Summary}

In conclusion for this section, power management schemes had shown better performance in-terms of reduced complexity levels and the contingent interference mitigation. Furthermore, relatively decreased exchanged interference between BSs can be achieved due to the reduction of the transmission power as well as boosted SINR levels and throughput achievements.

\section{GENERAL INTERFERENCE MANAGEMENT SCHEMES}

\section{A. Spectrum Management Schemes}

The authors of [34] presented an interference mitigation mechanism that combines spectrum management, coordination, and power management techniques together to allow multi non-subscribed users to be served by an FBS. Moreover, when the FBS determines the required spectrum that accommodates all users, the power reduction mechanism is activated. However, it degrades the system capacity despite the counter mechanism of calibrating both FBS gain and transmission power.

Alternatively, [35] proposed an algorithm for frequency allocation that determines all sub-channels that are available for Femto-cell usage. The authors further proposed an improved clustering algorithm to allow FUEs to use the entire spectrum dynamically. This helps to meet the requirements of the MUEs SINR. However, there are risks for the accessibility of the spectrum resources if high traffic occurs.

Moreover, the clustering algorithm divides the sub-channels among the available clusters and further allows each Femto-cell to access certain number of sub-channels to reduce the outage probability. Subsequently, it removes those with the greatest interference simultaneously and groups them together to form another cluster. Eventually, all Femto-cells are fairly allocated with the resources such that an improved spectral efficiency and a maximal reuse of resources will be achieved.

Similarly, [36] proposed general methods that are subject to the platform of the interference (cross-tier or cotier) such as interference management policy to optimize the performance of the network by managing the interference between FUEs. It determines the UEs that do not interfere to each other and allows them to transmit at the same time. Prior to that it calculates the threshold values which in return increase the network performance even in delay sensitive applications. 
Hence, the scheme computes an optimal threshold for the independent sets that can be scheduled together and proceeds with the scheduling. However, it is effective only if the distance between two BSs is below a certain limit.

\section{B. Fractional Frequency Reuse Schemes}

Fractional Frequency Reuse (FFR) is a widely known technique that partitions the bandwidth to reduce interference by allocating distinctive frequency portions to cell zones and further improves edge users performance [37] [38]. FFR increases the reuse factor as compared to the conventional technique by dividing the cell into regions and the bandwidth based on the requirements [39]. Furthermore, it has two branches, strict FFR which prevents center user from sharing the spectrum with edge users of all cells as shown in Figure 8a, while the soft FFR allows center users to share the resources with neighboring edge users as illustrated in Figure $8 \mathrm{~b}$.

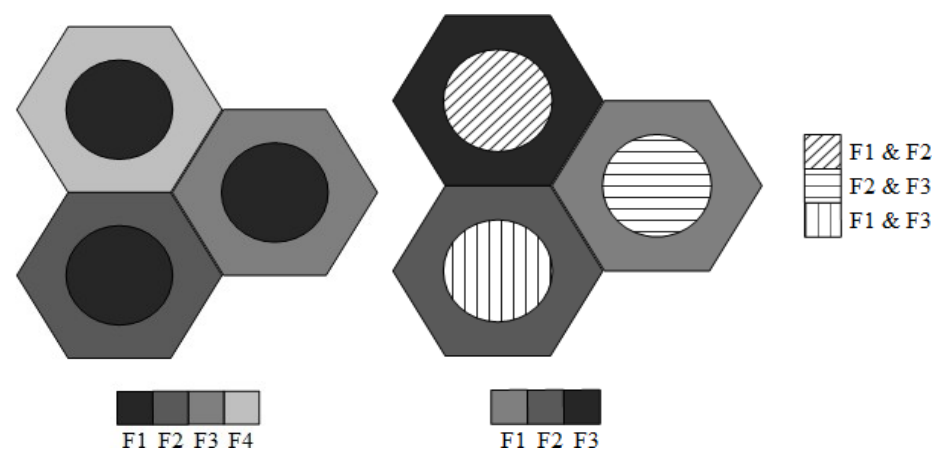

(a) Strict FFR.

(b) Soft FFR.

Fig. 8: Fractional Frequency Reuse branches [40].

Additionally, the authors in [40] in a prior study evaluated the network sum rate capacity for FFR branches as in Figure 9. Similarly, [41] proposed a novel FFR scheme that schedules the sub-carriers allocation at specific times resulting an improved spectral efficiency. Particularly, the scheme focuses on the cell edge users allowing them to contribute minimal interference which enables them to exploit the received interference for an easier mitigation. Additionally, the users minimize the transmission power and reduce their frequency reuse factor $\left.\left.1 /\left[3\left(\frac{2}{3}\right)+3\left(\frac{2}{3}\right)+\right)+3\left(\frac{2}{3}\right)+\right) / 7\right]=1.5$ rather than $\left.\left.1 /\left[3\left(\frac{1}{3}\right)+3\left(\frac{1}{3}\right)+\right)+3\left(\frac{1}{3}\right)+\right) / 7\right]=3$ in the traditional FFR scheme.

The authors of [42] presented a different method to reduce the frequency reuse factor by adjusting the transmission power of certain sub-bands. The scheme autonomously adjusts each sub-band independently in each sector of the Macro-cell which also reduces the interference and increases the throughput by up to $33 \%$. The scheme consists of two phases, the first uses the frequency hopping for averaging the interference, and the second phase collects feedback concerning the quality of the channel and SINR form via an indicator sent by the MUEs to the FBSs to improve the allocation process. Furthermore, it also indicates the MUEs transmission power, and carrier requirements in all sub-bands.

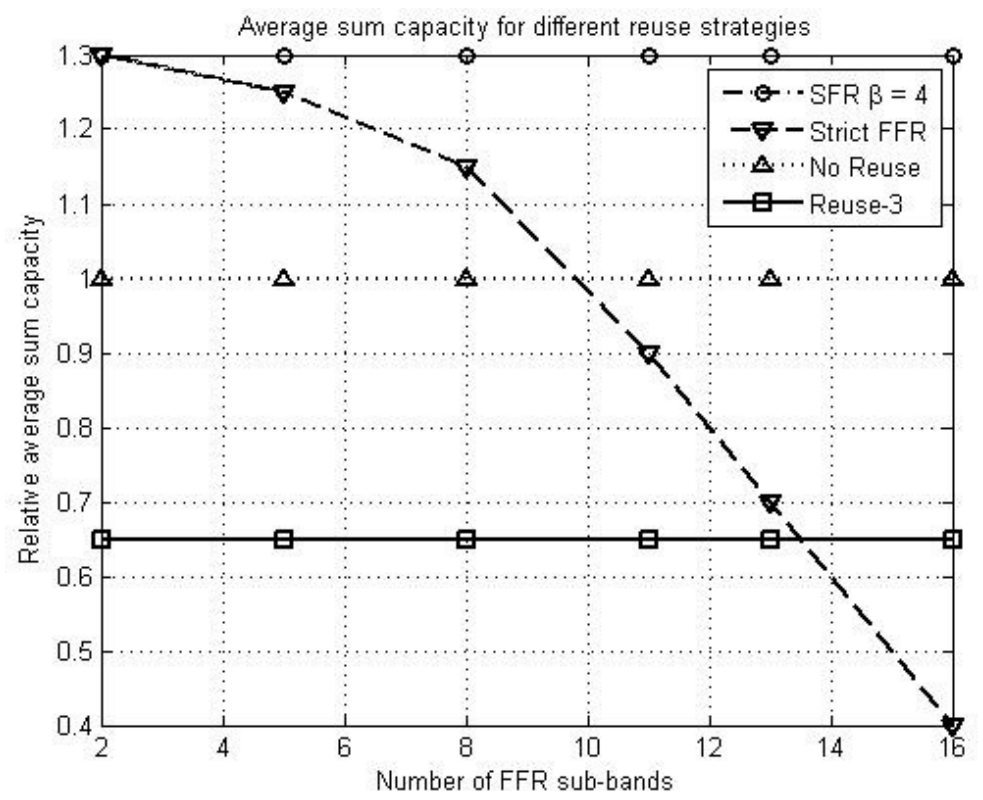

Fig. 9: Average sum rate capacity [40]. 
Moreover, a further publication of the same authors [43] scheduled the neighboring users to the sub-bands that experienced the transmission power suppression in aims to suppress the interference from the neighboring cells that use the same frequency for an easier and reliable management. It also restricts frequency hopping in between sub-carriers and rather allows it in between sub-bands. Consequently, the neighboring users experience only interference from the users who are on the same sub-bands which is quite similar to the conventional soft FFR. However, the authors reduced the power of the reused sub-bands resulting better throughput as compared to the prior design.

Additionally, [44] presented an improved version of the conventional FFR scheme by dividing the coverage area into inner region, outer region and three sectors. Furthermore, upon switching on a Femto-cell, the system registers the Femto-cell and marks it with a unique ID, as well as calculates the path-loss through the FBS location. It also allocates the spectrum based on the channel state information which is obtained by the following equation:

$$
\frac{C}{I}=\frac{P_{t 0} \times h_{0}}{\sum_{i} P_{r j} \times h_{j}+N_{0}}
$$

where $C$ is the power of the carrier, $I$ is the interference plus noise power, $h_{0}$ is the channel response and $P_{r j}$ is the received power.

Accordingly, the Femto-cell hops to the FUE second best channel when the FUE's SINR deteriorate below the threshold. With respect to that, in case of any problem during the hopping process, a Radio Network Controller (RNC) re-assigns the frequency to FBAa again. This is familiarly known as Hybrid Resource Management Algorithm (HRMA).

Upon evaluating the achieved system throughput presented in [45], it was concluded that the proposed system attained maximum of 115 Mbps for 180 FUEs while in [35], the system had achieved an approximate of 170 Mbps when deploying 100 FBSs and 130 Mbps when deploying 300 FBSs.

\section{Power Management Schemes}

As highlighted earlier, power leaks in general are the main factors behind interference in Femto-cell networks because of the close and random deployment. Hence, it is essential to calibrate the transmission power of both up-link and down-link in Femto-cells in-order to avoid interfering any nearby entity [46]. With regards to that, [47] employed a cooperative model that allowed Femto-cell to cooperatively mitigate the interference by adjusting the transmission power after calculating the received interference considering the path-loss models to insure high levels of user SINRs in both in-door and out-door transmissions.

Additionally, [48] proposed a dynamic power adaptation based on the traffic load and the FBS location scheme that insures the stability of FUEs and the QoS requirements. In return, the Femto-cell has to be capable of the utilizing frames and able to schedule the data transmission accordingly. This is to reduce the unnecessary mobility information which may increase delays. Moreover, the major elements of the frame utilization are the number of resource blocks that carry the data, the data being sent to the designated FUEs, and the path-loss between the FBS and the FUEs. Consequently, the power is accordingly adapted to a maximum limit whereby the same QoS is achieved.

Nevertheless, Femto-cells may also reduce the transmission power based on a Q-learning algorithm that is proposed in [49] which has two main branches. The first, partially distributes power allocation in between FBSs, while the second cooperatively distributes the power allocation. Consequently, each FBS is later awarded additional capacity figures upon quantizing the transmission power as the following:

$$
R=e^{-\left(C_{m}-B_{0}\right)^{2}}-e^{-C_{n}}
$$

where $C_{m}$ and $C_{n}$ are the aggregate capacity and $B_{0}$ is the targeted capacity.

The distributed power allocation differs from the prior as the Femto-cell information is shared with other FBSs to improve the overall performance.

In summary, [48] potentially reduced interference by adjusting the transmission power of FBSs while [46] had successfully mitigated interference in both down-link and up-link by calibrating the transmission power and carefully attenuating the up-link, respectively. Alternatively, the Q-learning method in [49] mitigated the interference, increased the capacity of the Femto-cell and MBS as well as stabilized network performance. However, only the SINR is maximized using the cooperative algorithm in [47].

\section{Interference Alignment and Coordination Schemes}

A decentralized interference coordination was proposed in [50] to mitigate the interference in both single tier and two-tier networks introducing a sub-carrier arbitrating algorithm. It allocates the least number of subcarriers to the BS through the following:

$$
N_{b} \leq N_{j}, j \in X_{b}
$$


where $N_{b}=\left|C_{b}\right|$ is the number of sub-carriers $C_{b}$ of cell $b, N_{j}=\left|C_{j}\right|$ is the number of sub-carriers of cell $j$ that is conflicting with cell $b$, and $X_{b}$ is the set of $j$ sub-carriers.

If Equation 20 is met, cell $b$ acquires the sub-carrier and conversely, cell $j$ acquires the sub-carriers. Accordingly, the coordination is done via choosing the sub-carrier that has the maximal SIR.

Generally, two-tier network scenarios provides different types of interference cases based on the medium platform.

1) between MBS and another adjacent MBS.

2) between MBS and another FBS of an adjacent MBS.

3) between FBS and another adjacent FBS of another MBS.

4) between MBS and its FBS.

5) between two FBSs of one MBS.

Hence, the coordination is performed via three stages as shown in Figure 10. Figure 10 (a) shows the available sub-carriers, 10 (b) shows the coordination of the first type of interference, 10 (c) is case $2 \& 3$ above, and $10(\mathrm{~d})$ is the last case.

The results of the scheme successfully reduced the interference as well as increased the utilization of subcarriers with an equal fairness level. However, the fairness level degrades due to the prioritization of BSs that possess the least number of sub-carriers which means that UEs count in the cell has no effect on the battle of sub-carriers. Consequently, large number BSs shares the resources unnecessarily due to unjust resource allocation in such scenarios. This will increase the interference level as the lack does not justify the demand in this scheme.

Alternatively, the authors of [51] proposed a scheme that allows $M U E s_{1,2,3, \ldots, j}$ to align their transmitted signals without causing any interference neither to other MUEs nor to an FBS. This is achieved by initially associating a precoder to MUEs to mitigate the FBSs up-link interference leakage which is calculated through Equation 21.

$$
\operatorname{SLNR} R_{j}=\frac{\left\|G_{j} z_{o j}\right\|_{F}^{2}}{N_{m} \sigma^{2}+\left\|G^{\prime} z_{o j}\right\|_{F}^{2}}
$$

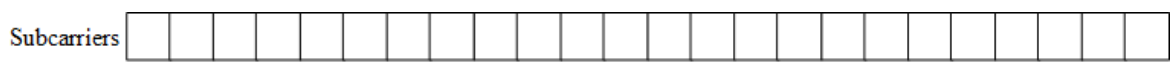

(a)

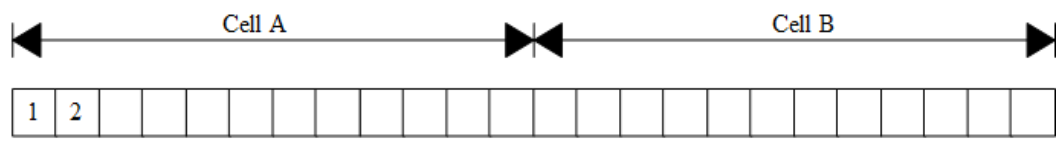

(b)

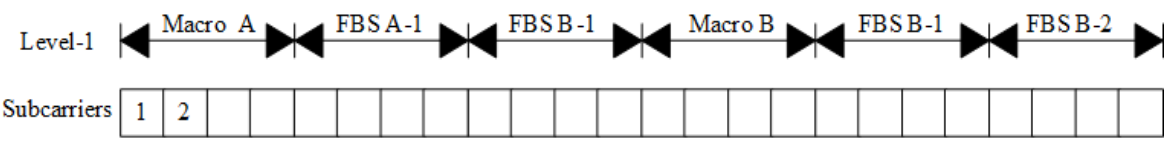

(c)

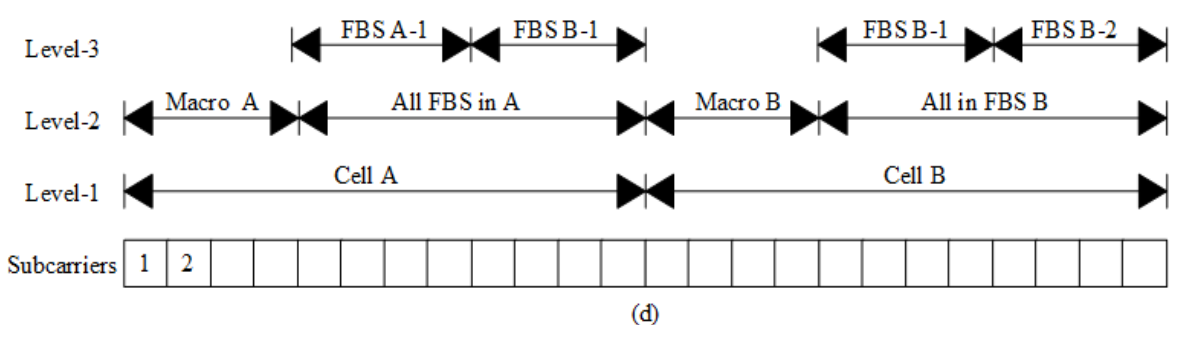

Fig. 10: Two tier interference coordination.

where $G_{j}$ is the channel with the interference alignment precoding matrix, $Z_{o j}$ is the second level precodêr and $G$ includes the interference from $j^{\text {th }}$ MUE to all FBSs.

It also calculates the coefficients of the precoder in a way that $M U E_{j}$ 's transmitted signals are mapped onto the subspace of all FBSs. Consequently, intensity of the leaked aligned interference is mitigated. 
In summary, [51] aligned the MUEs transmission power not to affect the Femto-cells, reduced BER, and improved FUEs' sum rate. Additionally, the method used in [50] had also mitigated the interference by increasing the sub-carrier utilization. However, this have resulted a reduction in fairness.

\section{E. Summary}

In conclusion of this section, it was shown that FFR schemes are easy to implement. It also offer high system performance, improved reliability and efficiency.

\section{CONCLUSION}

This paper provides a brief discussion on the problems and challenges concerning the interference in future Femto-cells networks. Additionally, it provides a comparison of the several contributions that have investigated the issue and presented some of the schemes and mechanisms that either avoid or reduce the interference. From the discussion in the co-tier scenarios, it can be summarized that power management schemes are more complex as compared to the spectrum management schemes due to the improved and reliable performance. However, power management schemes in cross-tier scenarios reduced the cross-tier interference and contributed to the cotier when decreasing the transmission power. Additionally, the FFR schemes in the last section can effectively mitigate both cross-tier and co-tier interference and improve the overall network performance of both the Marco-cell and Femto-cells.

\section{ACKNOWLEDGMENT}

The authors would like to acknowledge and appreciate the work provided by the authors of the papers listed in the reference section for the crucial and outstanding effort and contribution they offered to improve the cellular Femto-cell networks performance.

\section{REFERENCES}

[1] I. Poole, "Femtocell technology tutorial." Retrieved from http://www.radio electronics.com/info/cellulartelecomms/femtocells/ femtocells-network-architecture.php, 2012.

[2] G. Fanti and K. Femtocell networks." Retrieved https://pdfs.semanticscholar.org/bebd/ed478b7a7e852c9212d0dfa71922a0269791.pdf, 2012.

[3] E. Pateromichelakis, M. Shariat, A. U. Quddus, and R. Tafazolli, "On the analysis of co-tier interference in femtocells," in Personal Indoor and Mobile Radio Communications (PIMRC), 2011 IEEE 22nd International Symposium on, pp. 122-126, IEEE, 2011.

[4] K. C. Ting, H. C. Wang, C. C. Tseng, F. C. Kuo, and H. Te Huang, "Downlink co-tier interference mitigation for femtocell networks," in Intelligent Information Hiding and Multimedia Signal Processing (IIH-MSP), 2014 Tenth International Conference on, pp. 898-901, IEEE, 2014.

[5] B. Ma, M. H. Cheung, and V. W. Wong, "Interference management for multimedia femtocell networks with coalition formation game," in Communications (ICC), 2013 IEEE International Conference on, pp. 6112-6117, IEEE, 2013.

[6] R. N. Kummithe, "Interference mitigation in 4g lte-a heterogenous networks," master thesis, The University of Texas at Arlington, December 2012.

[7] S. Saleem, Developed mechanisms for cross-tier and co-tier interference avoidance in femtocell networks. PhD thesis, Victoria University, 2013.

[8] T. Zahir, K. Arshad, A. Nakata, and K. Moessner, "Interference management in femtocells," IEEE communications surveys \& tutorials, vol. 15, no. 1, pp. 293-311, 2013.

[9] M. Deruyck, W. Vereecken, W. Joseph, B. Lannoo, M. Pickavet, and L. Martens, "Reducing the power consumption in wireless access networks: Overview and recommendations," Progress In Electromagnetics Research, vol. 132, pp. 255-274, 2012.

[10] B. Ji, J. Zhu, K. Song, Y. Huang, and L. Yang, "Performance analysis of femtocells network with co-channel interference," Signal Processing, vol. 100, pp. 32-41, 2014.

[11] T. F. Collins and A. M. Wyglinski, "Co-channel interference in future femtocell networks," in Vehicular Technology Conference (VTC Fall), 2015 IEEE 82nd, pp. 1-2, IEEE, 2015.

[12] P. Palanisamy and S. Nirmala, "Downlink interference management in femtocell networks-a comprehensive study and survey," in Information Communication and Embedded Systems (ICICES), 2013 International Conference on, pp. 747-754, IEEE, 2013.

[13] K. Sireesha, T. S. Priya, and J. S. Babu, “Attenuation of co-channel interference in femtocell networks," International Journal of Applied Engineering Research, vol. 10, no. 3, pp. 8163-8172, 2015.

[14] T. Alade, H. Zhu, and J. Wang, "Uplink co-channel interference analysis and cancellation in femtocell based distributed antenna system," in Communications (ICC), 2010 IEEE International Conference on, pp. 1-5, IEEE, 2010.

[15] H. M. Mahbub, C. Alamgir, A. Rahman, S. Pal, and S. Shams, "Dynamic transmitter gain management approach for mitigating co-tier interference in femtocell network," in Telecommunication Networks and Applications Conference (ATNAC), 2014 Australasian, pp. 158-163, IEEE, 2014.

[16] V. Sathya, H. V. Gudivada, H. Narayanam, B. M. Krishna, and B. R. Tamma, "Enhanced distributed resource allocation and interference management in lte femtocell networks," in Wireless and Mobile Computing, Networking and Communications (WiMob), 2013 IEEE 9th International Conference on, pp. 553-558, IEEE, 2013.

[17] H.-C. Lee, D.-C. Oh, and Y.-H. Lee, "Mitigation of inter-femtocell interference with adaptive fractional frequency reuse," in Communications (ICC), 2010 IEEE International Conference on, pp. 1-5, IEEE, 2010.

[18] S. Khwandah, J. Cosmas, I. A. Glover, P. I. Lazaridis, G. Araniti, and Z. D. Zaharis, "An enhanced cognitive femtocell approach for co-channel downlink interference avoidance," IEEE Wireless Communications, vol. 23, no. 6, pp. 132-139, 2016.

[19] R. Raheem, A. Lasebae, M. Aiash, and J. Loo, "Interference management for co-channel mobile femtocells technology in lte networks," in Intelligent Environments (IE), 2016 12th International Conference on, pp. 80-87, IEEE, 2016.

[20] S. Saleem and H. King, "Avoidance of co-tier interference between femtocells with different access modes," International Journal of Information, vol. 2, no. 8, pp. 617-25, 2012.

[21] P. Liu, X. Wang, and J. Li, "Uplink co-tier interference management in femtocell networks with successive group decoding," IEEE Transactions on Wireless Communications, vol. 14, no. 9, pp. 4797-4809, 2015.

[22] M. E. Sahin, I. Guvenc, M.-R. Jeong, and H. Arslan, "Handling cci and ici in ofdma femtocell networks through frequency 
scheduling," IEEE Transactions on Consumer Electronics, vol. 55, no. 4, 2009.

[23] P. Vishnupriya, "Mitigation of co-channel interference in long term evolution," The International Journal of Engineering and Science (IJES), vol. 2, no. 4, pp. 2319-1805, 2013.

[24] N. Saquib, E. Hossain, and D. I. Kim, "Fractional frequency reuse for interference management in lte-advanced hetnets," IEEE Wireless Communications, vol. 20, no. 2, pp. 113-122, 2013.

[25] D. T. Ngo, L. B. Le, T. Le-Ngoc, E. Hossain, and D. I. Kim, "Distributed interference management in two-tier cdma femtocell networks," IEEE Transactions on Wireless Communications, vol. 11, no. 3, pp. 979-989, 2012.

[26] L. Hao, J. Ma, L. Zhao, H. Yang, and Z. Liu, "Joint power control and interference management in two-tier cdma femtocell networks for multi-agent systems," in Control Conference (CCC), 2014 33rd Chinese, pp. 1202-1207, IEEE, 2014.

[27] Q. Han, K. Ma, Z. Liu, and X. Guan, "Power control based on maximum power adaptation in two-tier femtocell networks," Wireless personal communications, pp. 1-21, 2013.

[28] H. Ren, C. Pan, W. Zhang, and M. Chen, "Downlink interference mitigation for ofdma femtocell networks," in Wireless Communications \& Signal Processing (WCSP), 2013 International Conference on, pp. 1-6, IEEE, 2013.

[29] M. M. Selim, M. El-Khamy, and M. El-Sharkawy, "Enhanced frequency reuse schemes for interference management in lte femtocell networks," in Wireless Communication Systems (ISWCS), 2012 International Symposium on, pp. 326-330, IEEE, 2012.

[30] D. Chen, T. Jiang, and Z. Zhang, "Frequency partitioning methods to mitigate cross-tier interference in two-tier femtocell networks," IEEE Transactions on Vehicular Technology, vol. 64, no. 5, pp. 1793-1805, 2015.

[31] V. N. Ha and L. B. Le, "Resource management for two-tier femtocell networks using interference alignment," in Globecom Workshops (GC Wkshps), 2013 IEEE, pp. 742-747, IEEE, 2013.

[32] B. Guler and A. Yener, "Uplink interference management for coexisting mimo femtocell and macrocell networks: An interference alignment approach," IEEE Transactions on Wireless Communications, vol. 13, no. 4, pp. 2246-2257, 2014.

[33] R. Ghaffar and P.-H. Ho, "Cross-tier interference mitigation in femto-macro cellular architecture in downlink," in Communications (ICC), 2013 IEEE International Conference on, pp. 5015-5020, IEEE, 2013.

[34] C. Bouras, G. Diles, V. Kokkinos, and A. Papazois, "Optimizing hybrid access femtocell clusters in 5g networks," in Broadband and Wireless Computing, Communication and Applications (BWCCA), 2015 10th International Conference on, pp. 220-226, IEEE, 2015.

[35] J. r. Shi and Q. Zhu, "Spectrum allocation based on interference management in femtocell networks," in International Conference on Automatic Control and Artificial Intelligence (ACAI 2012), pp. 1906-1909, March 2012.

[36] K. Ahuja, Y. Xiao, and M. van der Schaar, "Efficient interference management policies for femtocell networks," IEEE Transactions on Wireless Communications, vol. 14, no. 9, pp. 4879-4893, 2015.

[37] T. Lee, J. Yoon, S. Lee, and J. Shin, "Resource allocation analysis in ofdma femtocells using fractional frequency reuse," in Personal Indoor and Mobile Radio Communications (PIMRC), 2010 IEEE 21st International Symposium on, pp. 1224-1229, IEEE, 2010.

[38] P. Lee, T. Lee, J. Jeong, and J. Shin, "Interference management in lte femtocell systems using fractional frequency reuse," in Advanced Communication Technology (ICACT), 2010 The 12th International Conference on, vol. 2, pp. 1047-1051, IEEE, 2010.

[39] T. D. Novlan, R. K. Ganti, A. Ghosh, and J. G. Andrews, "Analytical evaluation of fractional frequency reuse for heterogeneous cellular networks," IEEE Transactions on Communications, vol. 60, no. 7, pp. 2029-2039, 2012.

[40] T. D. Novlan, R. K. Ganti, A. Ghosh, and J. G. Andrews, "Analytical evaluation of fractional frequency reuse for ofdma cellular networks," IEEE Transactions on wireless communications, vol. 10, no. 12, pp. 4294-4305, 2011.

[41] R. Ghaffar and R. Knopp, "Fractional frequency reuse and interference suppression for ofdma networks," in Modeling and Optimization in Mobile, Ad Hoc and Wireless Networks (WiOpt), 2010 Proceedings of the 8th International Symposium on, pp. 273277, IEEE, 2010.

[42] A. L. Stolyar and H. Viswanathan, "Self-organizing dynamic fractional frequency reuse in ofdma systems," in INFOCOM 2008. The 27th Conference on Computer Communications. IEEE, pp. 691-699, IEEE, 2008.

[43] A. L. Stolyar and H. Viswanathan, "Self-organizing dynamic fractional frequency reuse for best-effort traffic through distributed intercell coordination," in INFOCOM 2009, IEEE, pp. 1287-1295, IEEE, 2009.

[44] F. Tariq, L. S. Dooley, A. S. Poulton, and Y. Ji, "Dynamic fractional frequency reuse based hybrid resource management for femtocell networks," in Wireless Communications and Mobile Computing Conference (IWCMC), 2011 7th International, pp. 272-277, IEEE, 2011.

[45] T. Lee, J. Yoon, S. Lee, and J. Shin, "Resource allocation analysis in ofdma femtocells using fractional frequency reuse," in Personal Indoor and Mobile Radio Communications (PIMRC), 2010 IEEE 21st International Symposium on, pp. 1224-1229, IEEE, 2010.

[46] M. Yavuz, F. Meshkati, S. Nanda, A. Pokhariyal, N. Johnson, B. Raghothaman, and A. Richardson, "Interference management and performance analysis of umts/hspa+ femtocells," IEEE Communications Magazine, vol. 47, no. 9, pp. 102-109, 2009.

[47] A. Sanchez, J. Arauz, J. W. McClure, and Z. Miller, "Cooperative self-organized optimal power control for interference mitigation in femtocell networks," in Communications and Computing (COLCOM), 2016 IEEE Colombian Conference on, pp. 1-6, IEEE, 2016.

[48] P. Mach and Z. Becvar, "Dynamic power control mechanism for femtocells based on the frame utilization," in Wireless and Mobile Communications (ICWMC), 2010 6th International Conference on, pp. 498-503, IEEE, 2010.

[49] M. H. Elsayed and A. Mohamed, "Distributed interference management using q-learning in cognitive femtocell networks: new usrpbased implementation," in New Technologies, Mobility and Security (NTMS), 2015 7th International Conference on, pp. 1-5, IEEE, 2015.

[50] S. Wang, Y. Zhang, and G. Bi, "A decentralized interference coordination method for ofdma femtocell networks," in Wireless Communications \& Signal Processing (WCSP), 2012 International Conference on, pp. 1-5, IEEE, 2012.

[51] M. M. Gandha, S. Sameer, et al., "Two-level precoding based interference reduction in uplink for mimo femtocell networks," in Region 10 Conference (TENCON), 2016 IEEE, pp. 3034-3038, IEEE, 2016. 